\title{
Directed Attention and Perception of Temporal Order
}

\author{
Lew B. Stelmach \\ Communications Research Centre \\ Ottawa, Ontario, Canada
}

\author{
Chris M. Herdman \\ Carleton University \\ Ottawa, Ontario, Canada
}

\begin{abstract}
The present research examined the effects of directed attention on speed of information transmission in the visual system. Ss judged the temporal order of 2 stimuli while directing attention toward 1 of the stimuli or away from both stimuli. Perception of temporal order was influenced by directed attention: Given equal onset times, the attended stimulus appeared to occur before the unattended stimulus. Direction of attention also influenced the perception of simultaneity. The findings support the notion that attention affects the speed of transmission of information in the visual system. To account for the pattern of temporal order and simultaneity judgments, a model is proposed in which the temporal profile of visual responses is affected by directed attention.
\end{abstract}

In current models of directed attention, attention is assumed to influence the speed at which information is transmitted through the visual system. For example, in spotlight (or beam) (LaBerge, 1983; Posner, Snyder, \& Davidson, 1980) and zoom (Eriksen \& St. James, 1986; Eriksen \& Yeh, 1985) models, directing attention increases the transmission speed of visual information because the allocation of resources to the cued location increases. In gradient models (LaBerge \& Brown, 1989; Shaw, 1978), a gradient of attention that is centered and maximal at the attended location modulates the speed at which information is allowed to pass from a feature register to subsequent stages of processing in the perceptual system. Information flow is presumed to be greatest at the attended location because resources are abundant.

The purpose of the present research was to examine the assumption that attention modulates the speed of information transmission in the visual system. This was done by manipulating the direction of attention while requiring observers to judge the temporal order of two stimuli. We used the temporal-order task because it provides a sensitive index of information transmission speed (Sternberg \& Knoll, 1973; Ulrich, 1987).

Explicit manipulations of directed attention in tasks requiring temporal-order judgments have shown that the perception of temporal order may be influenced by attention when stimuli are presented in different sensory modalities (Sternberg, Knoll, \& Gates, 1971) or when stimuli are presented

This research was supported by the Communications Research Centre, Federal Government of Canada, and by two grants from the Natural Sciences and Engineering Research Council: one to Lew B. Stelmach and one to Chris M. Herdman. We thank Paul Hearty, Dorothy Phillips, and Vera Yuzyk for support throughout the course of this project. Fergus Craik, Vincent DiLollo, Peter Dixon, and Morris Moscovitch made helpful comments during the early stages of the project. Jo-Anne LeFevre provided a valuable critique of an earlier draft of the article.

Correspondence concerning this article should be addressed to Lew B. Stelmach, Communications Research Centre, 3701 Carling Avenue, Ottawa, Ontario, Canada K2H 8S2. Electronic mail: lew@ dgbt.doc.ca. within the auditory modality (Needham, 1936). In vision, there is indirect evidence suggesting that attention may influence the perception of temporal order of visual stimuli (Corwin \& Boynton, 1968; Sekuler, 1976; Sekuler, Tynan, \& Levinson, 1973). Corwin and Boynton (1968) found that when a foveal stimulus is presented simultaneously with a peripheral stimulus, the foveal stimulus appears to occur first (see also Rutschmann, 1966). In addition, Sekuler (Sekuler, 1976; Sekuler et al., 1973) found that left-of-center stimuli appear to occur before right-of-center stimuli. One possible explanation for these findings is that when left uncontrolled, attention is biased toward foveal or left-of-center locations, and that it enhances the speed of transmission of attended information. Because attention was not controlled in these studies, however, its role can only be inferred indirectly. In the present research, the direction of attention was manipulated explicitly.

The theoretical framework used in the present research is based on an integration of the general threshold model of temporal-order judgments (Ulrich, 1987) with current models of directed attention. The general threshold model was used because it subsumes several extant models of temporal-order judgments (e.g., Allan, 1975; Sternberg \& Knoll, 1973). According to the general threshold model, temporal-order judgments depend on the arrival time of visual responses at a temporal comparator. Differences in arrival order of two stimuli are perceived when the arrival times of the stimuli are separated by a minimum duration, referred to as $C_{x y}$ (Ulrich, 1987): $C_{x y}$ may be regarded as a refractory period of the comparator. If two sensory messages arrive in fast succession and are separated by less than $\mathrm{C}_{x y}$, the comparator cannot determine their arrival order, and simultaneity is perceived. Arrival of two stimuli at the temporal comparator depends on the relative onset times of the stimuli and the transmission latencies of the sensory signals from the retina to the temporal comparator. Onset times of stimuli depend on display parameters, whereas latencies depend on properties of the sensory pathways.

In accordance with the spotlight (LaBerge, 1983; Posner et al., 1980), zoom (Eriksen \& Yeh, 1985) and gradient (LaBerge \& Brown, 1989) models of directed attention, attention can 
be assumed to affect the transmission latencies of visual responses from the retina to the temporal comparator. In this view, attended stimuli should reach the temporal comparator with a shorter latency than unattended stimuli because the speed of transmission through the perceptual system is greater for attended information. Consequently, if two stimuli are presented at the same time (stimulus onset asynchrony [SOA] $=0 \mathrm{~ms}$ ), observers should perceive that an attended stimulus occurs before an unattended stimulus. In general, perceived temporal order (left first or right first) at an SOA of $0 \mathrm{~ms}$ should depend on whether the observer directs attention the left or to the right, respectively. If both stimulus locations receive equal amounts of attention, then the transmission latencies of the stimuli should be the same, and arrival order at the temporal comparator will depend completely on display timing.

In six experiments, we examined the assumption that attention affects the speed of information transmission in vision. Experiments 1 and 2 establish the effects of directed attention on the perception of temporal order. Experiment 3 shows that these effects cannot be attributed to eye movements. Experiments 4-6 address some methodological issues and extend the basic findings by examining the effect of directed attention on perceived simultaneity.

\section{Experiment 1}

In Experiment 1, we examined the effects of directed attention on the perception of temporal order through the use of a computerized parameter estimation technique (PEST; see Taylor \& Creelman, 1967). Observers were required to fixate centrally and direct attention either to the left (left attend), to the right (right attend), or to the center (center attend). On each trial, two brief flashes of light were presented, one to the left of fixation and the other to the right of fixation. Observers were required to indicate which light appeared first. PEST adjusted the SOA until each stimulus was selected approximately $50 \%$ of the time, that is, until observers could no longer discriminate which stimulus came on first. The SOA at which this occurs is referred to as the point of greatest temporal uncertainty. The display sequence is illustrated schematically in Figure 1.

The temporal order of the two stimuli should become indiscriminable to the observer when the sensory signals corresponding to the occurrence of the stimuli reach the temporal comparator at about the same time. When attention is directed centrally (equidistant from the left and right stimulus locations), an equal amount of attention is allocated to both stimulus locations. In the center-attend condition the point of greatest temporal uncertainty should occur at an SOA of $0 \mathrm{~ms}$ because the transmission latencies of the sensory signals should be equivalent and the arrival order of the signals at the temporal comparator should be the same. When attention is directed to either of the two stimulus locations (left or right), transmission latencies should differ. Specifically, given equal onset times $(\mathrm{SOA}=0 \mathrm{~ms}$ ), the stimulus presented at the attended location should reach the temporal comparator before the stimulus presented at the unattended location. Consequently, the observer should perceive the attended stim-

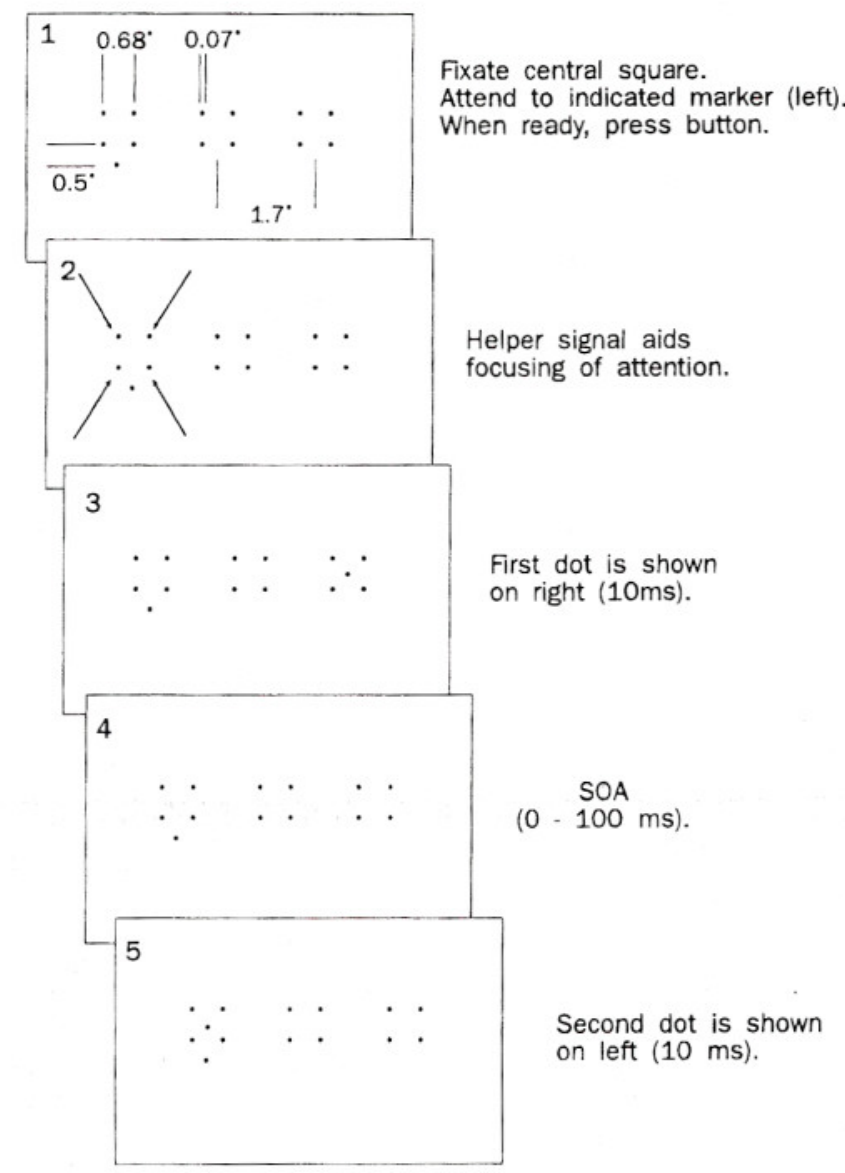

Figure 1. Schematic representation of the display sequence.

ulus as occurring first. The point of greatest temporal uncertainty would occur when the unattended stimulus physically precedes the attended stimulus by some SOA. For example, when attention is directed to the right of fixation, the point of greatest temporal uncertainty would occur when the stimulus to the left of fixation is displayed first by some SOA. Similarly, when attention is directed to the left of fixation, the point of greatest temporal uncertainty would occur when the stimulus to the right of fixation is displayed first by some SOA. However, if attention does not affect transmission latencies of sensory signals to the temporal comparator, then directing attention to the left or right stimulus locations should not influence temporal-order judgments. That is, the point of greatest temporal uncertainty should be at an SOA of $0 \mathrm{~ms}$.

\section{Method}

Observers. Nine observers participated in the experiment, including the 2 authors and 7 observers who were unaware of the hypothesis under investigation.

Display. The display was presented on an HP1340 oscilloscope equipped with P4 phosphor and controlled by a Digital Equipment PDP 11/73 computer. There were five parts to the display, as shown in Figure 1. Part 1 consisted of three location markers and an indicator dot below one of the markers. The indicator dot identified the location to which attention was to be directed. Each marker was assembled 
from four dots arranged in a square of side $0.68^{\circ}$. The center-tocenter distance between adjacent markers was $1.7^{\circ}$. Part 1 was visible throughout the trial. Other parts were superimposed on Part 1, as shown in Figure 1.

The observer initiated Part 2 of the display by pressing a button. Part 2 consisted of a visual "helper signal" designed to aid observers in directing their attention to the indicated position. The helper signal was composed of four dots that moved from the outer portions of the screen to the corners of the marker in $1 \mathrm{~s}$. At the starting position of the helper signal, the distance between each dot was $7.0^{\circ}$, as measured along the horizontal and vertical axes. After the helper signal had ended, there was a $250-\mathrm{ms}$ pause during which the display was identical to Part 1.

Part 3 of the display consisted of a stimulus dot within the left or right marker (Figure 1 shows the dot in the marker on the right). The dot was shown for $10 \mathrm{~ms}$ at a brightness comfortably above threshold. Background luminance was $89 \mathrm{~cd} / \mathrm{m}^{2}$. Part 4 of the display consisted of the SOA. Part 5 consisted of the second stimulus dot displayed for $10 \mathrm{~ms}$. In Figure 1, the second dot is shown on the left. Note that at an SOA of 0 ms Parts 3 and 5 were displayed simultaneously.

Design. There were six conditions in the experiment, which were defined by the factorial combination of three loci of attention (left, center, and right) and two starting SOAs $(70$ and -70$)$. A positive SOA was arbitrarily assigned to situations in which the right dot was presented before the left dot. Consequently, a negative SOA indicates that the left dot was presented before the right dot. Each observer served in all conditions of the experiment.

Procedure. A two-alternative forced-choice procedure was used. On each trial, the observer fixated on the central marker and directed attention to the indicated marker (left, right, or center). When ready, the observer pressed a button to initiate the trial. The trial ended when the observer indicated which stimulus dot appeared first, again by pressing a button.

During each session, the PEST program made six estimates of the point of greatest temporal uncertainty, one for each of the six conditions of the experiment ( 3 attentional conditions $\times 2$ starting SOAs). The estimates were made in parallel; that is, at the beginning of every session the initial SOAs for each attentional condition were set at two levels (70 $\mathrm{ms}$ and $-70 \mathrm{~ms}$ ). As the session progressed, the PEST program monitored the observer's responses and adaptively adjusted each SOA in search of the point of greatest temporal uncertainty. At the end of each session, PEST output six numbers (one for each of the six conditions) corresponding to the critical SOA at which observers could not differentiate the order of the two stimuli.

Conditions were tested in a random order (i.e., mixed blocks) such that observers could not predict the attentional condition from one trial to the next. The positive and negative starting SOAs were used to ensure that each attentional condition was not reliably associated with a left-first or a right-first stimulus sequence. A session lasted about $20 \mathrm{~min}$. Each observer served in six sessions.

Instructions. Observers were instructed to fixate on the central marker and direct their attention to the indicated marker prior to pressing the start button and to use the helper signal to assist in focusing their attention on the marker. They were instructed not to make eye movements from the time they pressed the start button until the two stimulus dots had been displayed. Observers were told to respond at their own pace and to select which stimulus appeared first. In those cases in which they were uncertain, observers were told to avoid guessing but to base their choice on the perceptual evidence that was available.

\section{Results and Discussion}

Results are shown in Figure 2 with histograms. The histograms should not be confused with the line graphs because the latter show the results of Experiment 2. The histogram in each panel shows the frequency (summed across subjects, sessions, and starting SOA) with which PEST estimated each SOA to be the point of greatest temporal uncertainty. We constructed the histograms by first quantizing the SOA range into 20 -ms bins and then plotting the frequency with which the estimates fell into each bin as a percentage of the total.

The data were analyzed with a 3 (attentional locus: left, right, or center) $\times 2$ (starting SOA: $70 \mathrm{~ms}$ and $-70 \mathrm{~ms}) \times 6$ (session) analysis of variance (ANOVA). The hypothesis that the point of greatest temporal uncertainty is affected by direction of attention was supported by a significant main effect of attentional locus, $F(2,16)=118.85, p<.001$. As predicted, in the center-attend condition (Figure 2, bottom panel), temporal order was indiscriminable at an SOA close to $0 \mathrm{~ms}$. Therefore, when attention was directed equally to both stimulus locations there is no evidence that transmission times differed. Importantly, in the left-attend condition (Figure 2, center panel), temporal order was indiscriminable when the right (unattended) stimulus preceded the left (attended) stimulus by about $40 \mathrm{~ms}$. Similarly, in the right-attend condition (Figure 2, top panel), temporal order was indiscriminable when the left (unattended) stimulus preceded the right (attended) stimulus by about $40 \mathrm{~ms}$. The effects in the left-
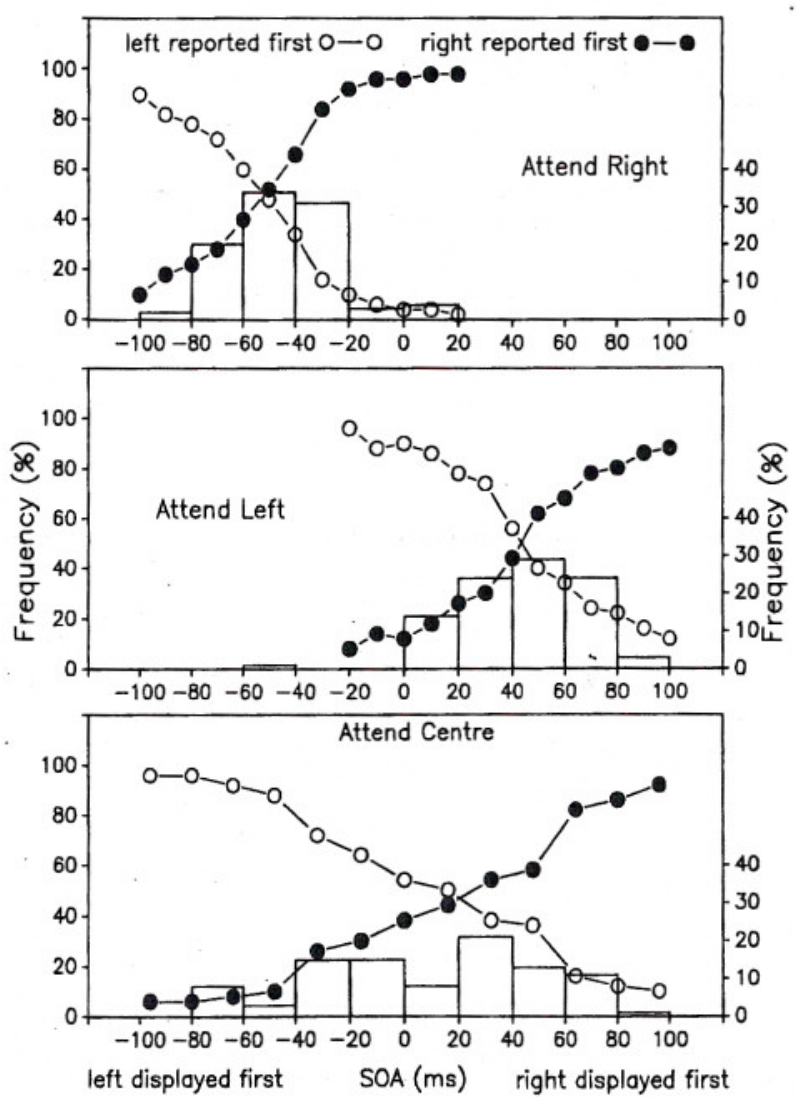

Figure 2. Histograms (Experiment 1) show the frequency with which PEST estimated the point of greatest temporal uncertainty at a given SOA; line graphs (Experiment 2) show the frequency with which observers responded left first and right first at a given SOA. 
and right-attend conditions are consistent with the notion that attention shortens the transmission latency of signals from the retina to the temporal comparator. For the unattended and attended stimuli to arrive at the temporal comparator at the same time, the unattended stimulus had to be displayed about $40 \mathrm{~ms}$ in advance of the attended stimulus.

There was also a main effect of starting SOA, $F(1,8)=$ 9.26, $p<.02$ : For each attentional condition, there was a range of SOAs at which observers were uncertain about the order of the two stimuli. The starting SOA biased PEST to one or the other end of this range. Thus, the left half of each histogram consists predominantly of estimates from conditions in which the starting SOA was $-70 \mathrm{~ms}$. The right half of each histogram consists predominantly of estimates from conditions in which the starting SOA was $70 \mathrm{~ms}$. For this reason, the mean of each histogram is as an unbiased estimate of the center of this range.

Figure 2 shows that the dispersion of the histogram for the center-attend condition was greater than that of the other two attentional conditions, as is reflected in a significant Starting SOA $\times$ Attentional Locus interaction, $F(2,16)=9.68, p<$ .002 . Thus the range of SOAs at which observers were uncertain about the temporal order of the two stimuli was greater in the center-attend condition. The effects of session and all interactions involving session were not statistically significant $(F \mathrm{~S}<1)$.

To summarize, in the left- and right-attend conditions, the temporal order of the stimuli appeared to be indiscriminable when the stimulus at the unattended location led the stimulus at the attended location by approximately $40 \mathrm{~ms}$. These findings suggest that information about the attended stimulus reached the temporal comparator with a shorter latency than information about the unattended stimulus. In accordance with extant models of directed attention and with the general threshold model, the results can be explained by assuming that en route to the temporal comparator, attention speeds up the transmission of information in the visual system. Note, however, that changing the arrival order of the sensory signals should not change the dispersion of the distributions but should only shift their relative positions. The differences in dispersion may be explained within the general threshold model (Ulrich, 1987) by assuming that $\mathrm{C}_{x y}$, the refractory period of the temporal comparator, is shorter when attention is directed to one of the stimuli, as occurred in the left- and right-attend conditions. According to this interpretation, the dispersion of the response distributions (Figure 2) indexes the duration of $\mathrm{C}_{x y}$ : A shorter refractory period produces less dispersion (as in the left- and right-attend conditions) than a longer refractory period (as in the center-attend condition).

\section{Experiment 2}

The PEST technique was used in Experiment 1 to estimate points of greatest uncertainty about temporal order (i.e., the SOA at which an observer selected the left and right stimuli with equal frequency). The second experiment was designed to provide a converging estimate of these points and to characterize further the function relating frequency of selection (left first or right first) to SOA. To do this, we estimated the psychometric function relating temporal-order judgments to SOA over the full range $(0 \%-100 \%$ of left-first and rightfirst responses) by using the method of constant stimuli.

\section{Method}

Observers. Six observers participated in the experiment, the 2 authors and 4 others who were unaware of the hypothesis under investigation. All had participated in the previous experiment.

Design. There were 2 variables in the experiment: locus of attention (left, center, and right) and SOA at 13 levels, for a total of 39 conditions. In the center-attend condition SOAs ranged (in steps of $16 \mathrm{~ms}$ ) from $-96 \mathrm{~ms}$ to $96 \mathrm{~ms}$. In the left- and right-attend conditions SOAs ranged (in steps of $10 \mathrm{~ms}$ ) from $-20 \mathrm{~ms}$ to $100 \mathrm{~ms}$ and from $-100 \mathrm{~ms}$ to $20 \mathrm{~ms}$, respectively. Each range of SOAs was selected to measure the complete psychometric function (i.e., from $0 \%$ to $100 \%$ selected left first and right first). Different step sizes were used in the center-attend versus the left- and right-attend conditions to equate the number of sampling points on the psychometric functions. Each observer served in all conditions of the experiment. Conditions were tested in random order (i.e., mixed blocks) such that observers could not predict the attentional condition or the temporal order from trial to trial.

Procedures. The display was identical to that used in Experiment 1 (see Figure 1). Procedures were the same as in Experiment 1, with the following exceptions. The SOA was selected randomly on each trial rather than being adjusted adaptively by PEST. Each observer was tested 50 times in each condition over the course of 10 sessions. During each session all 39 conditions ( 3 Attentional Loci $\times 13$ SOAs) were tested five times each in random order. A session lasted approximately $25 \mathrm{~min}$.

\section{Results and Discussion}

Results are shown with line graphs in Figure 2. Each point represents the percentage of left-first (open points) and rightfirst (solid points) judgments at a given SOA averaged across all observers.

The results were analyzed in two ways. First, we compared the crossover points of the curves for the three attentional conditions. The crossover points identify the SOA at which observers could not tell the temporal order of the two stimuli and were equally likely to respond left first or right first. For each observer, three crossover points were calculated (one for each of the three attentional conditions). A one-way repeated measures ANOVA showed that crossover points differed for the three conditions, $F(2,10)=42.01, p<.001$. The mean crossover point was near an SOA of $0 \mathrm{~ms}$ only in the centerattend condition, as is expected if attention was approximately equally distributed at the two stimulus locations. In both leftand right-attend conditions, at an SOA of $0 \mathrm{~ms}$ the attended stimulus was judged to come on first virtually $100 \%$ of the time. Temporal order of the stimuli was indiscriminable in the left- and right-attend conditions when the unattended stimulus preceded the attended stimulus by an average of 56 and $44 \mathrm{~ms}$, respectively. These results are consistent with those of Experiment 1 and support the hypothesis that attention increases the speed at which information is transmitted in the visual system.

In a second analysis, we compared the slopes of the curves of the three attentional conditions (Figure 2). Slopes were 
calculated separately for each observer for each attentional condition in two different ways. In one, a linear function was fit to the nonasymptotic portion of each subject's curve. In the other, a logistic function was fit to the entire curve for each subject. In the logistic function, Formula (1), parameter $a$ is the central tendency and parameter $b$ is the slope. Note that for the linear function a larger value indicates a steeper slope, whereas for the logistic function a smaller value indicates a steeper slope.

$$
f(x, a, b)=1 /(1+\exp (-(x-a) / b)) .
$$

The linear and logistic methods for calculating the slope yielded similar conclusions. Separate one-way repeated measures ANOVAs showed that slopes differed significantly in the three attentional conditions, $F(2,10)=9.35, p<.005$ (linear function); $F(2,10)=5.38, p<.03$ (logistic function). Mean values for the slopes were $0.88,0.70$, and 0.35 (linear) and $24,16.3$, and 34.9 (logistic) for the left-, right-, and centerattend conditions. The differences in slopes across attentional conditions are consistent with the dispersion of the histograms from Experiment 1 (see Figure 2). The shallower slope of the curve in the center-attend condition is associated with a histogram that has a greater dispersion and indicates that there was a larger range of SOAs over which observers were uncertain about the temporal order of the stimuli. The steeper slopes of the curves in the left- and right-attend conditions are associated with histograms that have lesser dispersions. As explained before, the general threshold model (Ulrich, 1987) may be modified to account for the differences in dispersions among the conditions by including the assumption that the temporal comparator has a shorter refractory period in the left- and right-attend conditions.

\section{Experiment 3}

In Experiments 1 and 2 observers were instructed to maintain fixation on the center square throughout each trial. Although eye movements were not monitored, it was relatively easy for subjects to maintain fixation after some practice. Nevertheless, it was important to demonstrate that the results of the previous experiments did not arise from eye movements that could lead to fixation of the attended stimulus dot. In the present experiment, trials on which fixation deviated to the left or to the right of the central (fixation) marker were discarded and repeated later in the session.

\section{Method}

Observers. Six observers participated in the experiment, the 2 authors and 4 observers who were unaware of the hypothesis under investigation. All but 1 of the observers had participated in the previous experiments.

Apparatus and calibration. An ISCAN RK-416 pupil-tracking system with noise-reduction software and eye magnification optics was used to monitor eye position. Head movements were minimized through the use of a head restraint and chinrest.

As in the previous experiments, the distance from the center of the central fixation marker to either edge of the marker was $0.35^{\circ}$. To ensure that fixation did not deviate beyond the left or right edge of the central marker, the pupil-tracking system was set up to detect horizontal eye movements of amplitude greater than $0.26^{\circ}$ of visual angle. Note that this is a relatively stringent criterion, which rejects eye movements that approach either the left or the right side of the central marker.

The horizontal sensitivity of the pupil-tracking system was measured both with an artificial eye (diameter $25 \mathrm{~mm}$, pupil size $3 \mathrm{~mm}$ ) and with human subjects. In both cases, the response of the pupiltracking system was recorded by using $0.25^{\circ}$ steps: The artificial eye was moved manually in the horizontal direction in steps of $0.25^{\circ}$, and the human observers were instructed to stare at a cross hair that moved horizontally in steps of $0.25^{\circ}$.

The results of the calibration (Figure 3) show that there was agreement between the artificial and human calibration data. A $0.25^{\circ}$ movement of the eye (human or artificial) generated a 3.9-unit response, on average, from the pupil-tracking system. A linear fit to the curve in Figure 3, with intercept fixed at 0, yielded a slope of 15.44. The horizontal resolution of the pupil-tracking system, corresponding to a 1-unit change in its response, was $0.065^{\circ}$, or about 4 min of arc. This calibration demonstrates that the pupil-tracking system is easily capable of detecting the requisite $0.26^{\circ}$ (4-unit) shift in eye position.

Design and procedure. Subjects were tested in two stages. The first, without pupil monitoring, was intended to establish a baseline, and the second, with pupil monitoring, was intended to control for inappropriate eye movements. In both stages (baseline and pupil monitoring), the display, design, and procedures were identical to those of Experiment 2 except that each observer participated in 6 rather than 10 sessions. This yielded 30 observations per condition per subject. In the pupil-monitoring stage, the validity of a trial was determined by comparing the pupil position at the start of each trial (during initial fixation) with the pupil position during the presentation of the two stimulus dots. If the pupil had moved more than $0.26^{\circ}$ horizontally, the trial was deemed invalid and repeated later in the session.

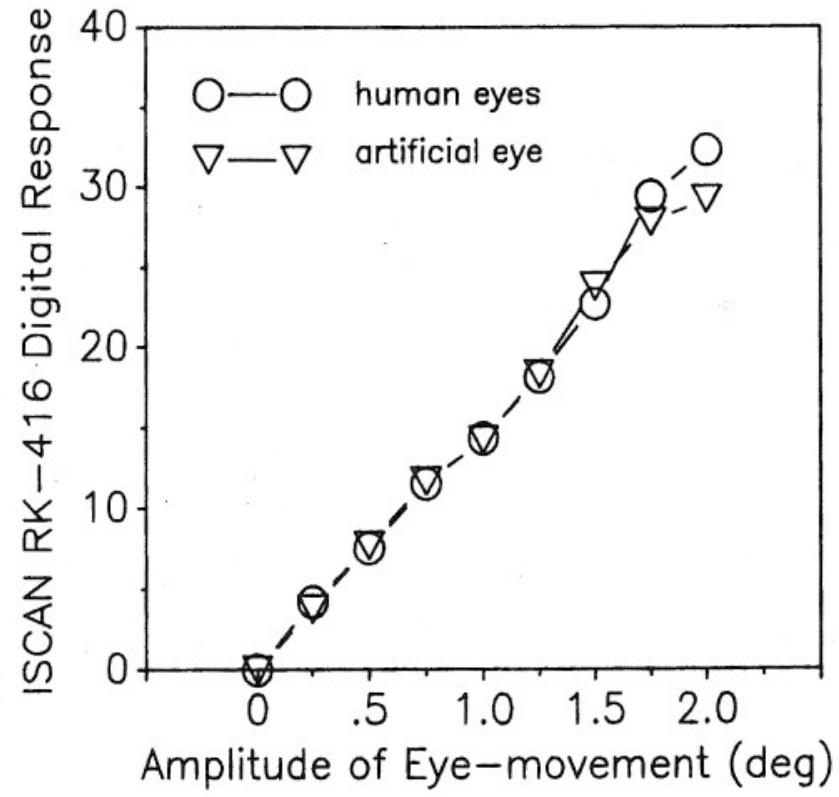

Figure 3. Digital response of the pupil-monitoring system to horizontal movements of an artificial eye and of human eyes. 


\section{Results and Discussion}

An average of 57.8 trials was repeated in each session of the pupil-monitoring stage. This value was somewhat inflated by 1 subject who had an average of 134 trials repeated per session. The mean number of trials repeated per session for the other 5 subjects was 35 . Eye movements that gave rise to the repeated trials (for all 6 subjects) had a mean amplitude of $0.47^{\circ}$. The amplitude indicates that most of the inappropriate eye movements were just beyond (approximately $0.12^{\circ}$ ) an edge of the central fixation marker. The repetition rate and the amplitude of the inappropriate eye movements did not differ among the three attentional conditions. That is, an inappropriate eye movement was equally likely to occur in the left-, right-, and center-attend conditions.

In the left- and right-attend conditions, $52 \%$ of the eye movements were in the direction of the attended side and $48 \%$ were in the direction of the unattended side. Thus, any eye movements that did occur represent a failure to maintain steady fixation; they do not represent a systematic bias to look in the direction of the attended side.

The results from the baseline and pupil-monitoring stages of the experiment are shown in Figure 4 with solid and dashed lines, respectively. As Figure 4 clearly shows, there was close agreement between the results of both stages. Therefore, we conclude that directed attention and not changes in fixation was responsible for the pattern of results.

\section{Experiment 4}

The purpose of Experiments 4 and 5 was to rule out the possibility that the previous results were influenced by constraints of the two-alternative forced-choice procedure. Specifically, because a simultaneous response alternative was not available in Experiments 1-3, it is possible that on trials when the stimuli were perceived to occur simultaneously, observers were biased to respond right first in the right-attend condition and left first in the left-attend condition. This may explain why the crossover points and the histograms were shifted away from $0 \mathrm{~ms}$ in the left- and right-attend conditions.' The possibility that observers were allocating simultaneous perceptions in this manner was investigated in the present experiment by introducing a simultaneous-response alternative. If the differences among the three attentional conditions evident in Figures 2 and 4 were caused by the allocation of simultaneous perceptions to the left-first and right-first responses, then the availability of the simultaneous response ought to eliminate differences between the three attentional conditions.

On the other hand, if the attentional effects persist, then the pattern of simultaneous responses may provide additional information concerning the effects of directed attention on visual processing. According to the general threshold model (Ulrich, 1987), simultaneous responses should occur when the sensory signals arrive within the refractory period of the temporal comparator $\left(\mathrm{C}_{x y}\right)$. This should correspond to the point of greatest temporal uncertainty identified in the previous experiments (about $-40,0$, and $40 \mathrm{~ms}$ in the right-, center-, and left-attend conditions, respectively). In each attentional condition, simultaneous responses should be distrib-
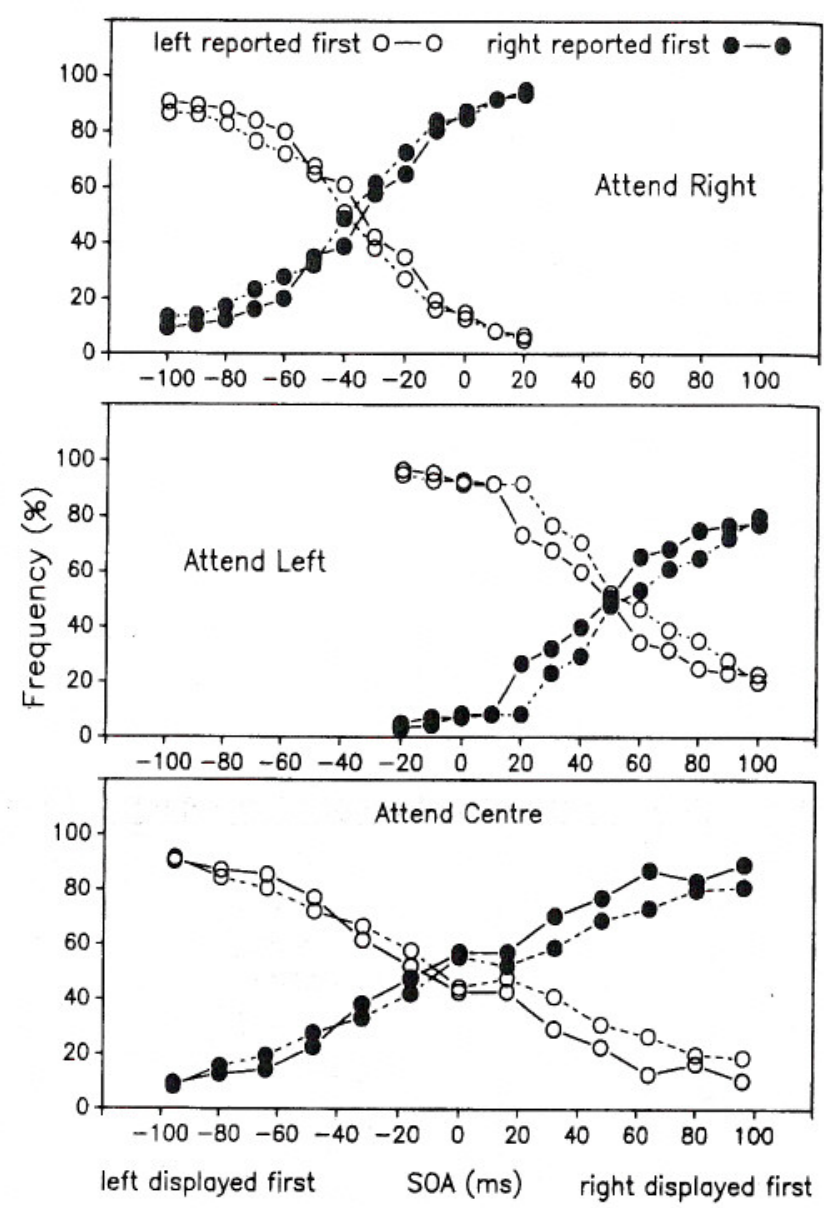

Figure 4. Frequency of left-first and right-first responses (Experiment 3 ) across SOAs when eye movements were monitored (dashed lines) and not monitored (solid lines).

uted around the SOA of greatest temporal uncertainty. There ought to be a concomitant decline in the frequency of leftfirst and right-first responses at these SOAs.

\section{Method}

Observers. Five observers participated in the experiment, the 2 authors and 3 others who were unaware of the hypothesis under investigation. All observers had participated in at least two of the previous experiments.

Design and procedure. The display and design were identical to that used in Experiment 2. Procedures were the same as in Experiment 2 except that observers had three rather than two response alternatives: left first, right first, or simultaneous. Observers were instructed to select the simultaneous-response alternative only when the two dots were perceived to occur simultaneously and to select one of the other two response alternatives when the dots were perceived to be asynchronous.

\footnotetext{
'In this view, a shallower slope may have occurred in the centerattend condition because simultaneous perceptions would be divided between two response alternatives, whereas in the left- and rightattend conditions they would be allocated to one response alternative.
} 


\section{Results and Discussion}

Results are shown in Figure 5. Each point represents the percentage of left-first (open points), right-first (solid points), and simultaneous (open triangles) judgments at a given SOA, averaged across all observers.

According to the general threshold model, simultaneous responses should be most frequent at points of greatest temporal uncertainty. This prediction was supported in the center-attend condition. As can be seen in Figure 5 (bottom panel), simultaneous responses were centered on and evenly distributed around an SOA of 0 ms. As the absolute SOA increased, the proportion of left-first and right-first judgments increased. In marked contrast to the relatively large number of simultaneous responses in the center-attend condition ( $35 \%$ of total responses), there were very few simultaneous responses in the left- and right-attend conditions (4\% of total responses in each condition). This pattern of results does not agree with the predictions of the general threshold model: Observers did not perceive simultaneity at the point of greatest temporal uncertainty. In the general threshold model, temporal uncertainty and simultaneity are interchangeable; both reflect the case in which the sensory signals arrive within the refractory period of the temporal comparator $\left(\mathrm{C}_{x y}\right)$.

It might be argued that the duration of $\mathrm{C}_{x y}$ was shorter in the left- and right-attend conditions and that simultaneity is

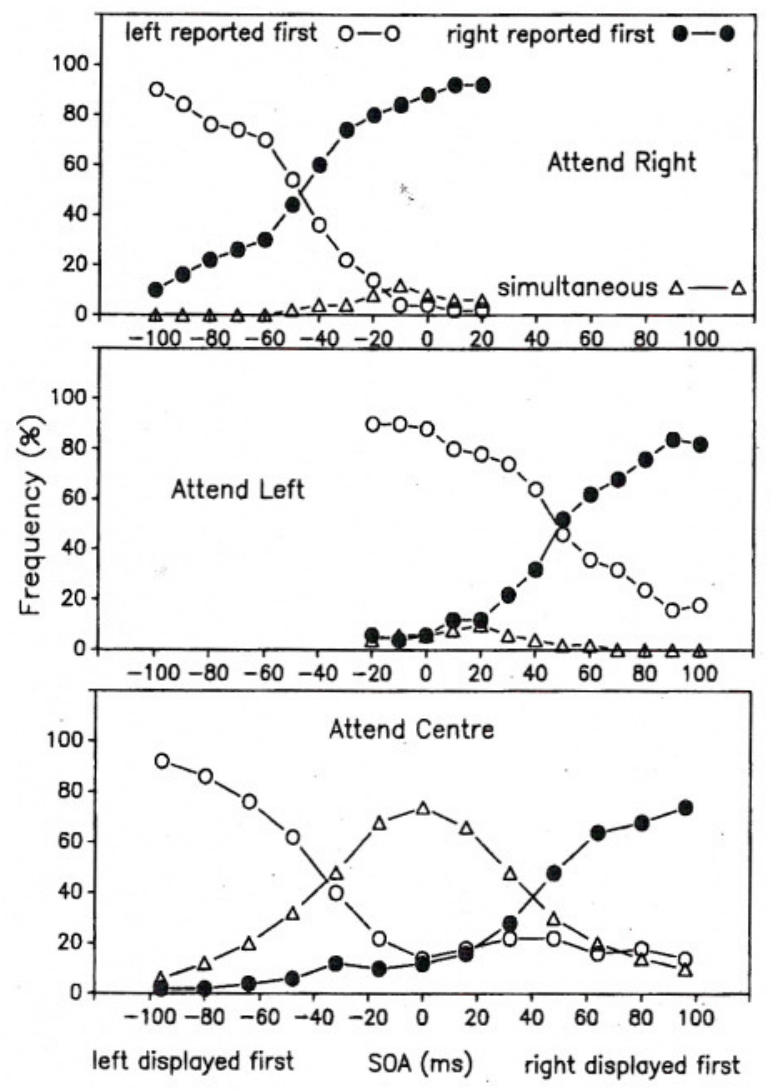

Figure 5. Frequency of left-first, right-first, and simultaneous responses across SOAs (Experiment 4). perceived over a narrower range of SOAs in these conditions than in the center-attend condition. The range of temporal uncertainty in the left- and right-attend conditions, however, suggests that the duration of $\mathrm{C}_{x y}$ was still substantial and that a distribution of simultaneous responses centered on this range would still be expected. Therefore, it seems that the general threshold model does not account for all aspects of the present data. An alternative model that can account for the virtual absence of simultaneous responses at the point of greatest temporal uncertainty is presented in the General Discussion section.

\section{Experiment 5}

Experiment 4 rejects one version of a response-artifact explanation dealing with the allocation of simultaneous perceptions to the left and right response alternatives; however, another version remains tenable. It could be argued that adding a simultaneous-response alternative did not completely redress the limitations of the two-alternative forcedchoice procedure. One possibility is that observers may have been confident that the stimuli did not occur simultaneously but were not sure whether the left or the right stimulus came on first. In this situation, they may have selected by default the response corresponding to the side to which they were attending. As discussed earlier, a response strategy of this sort. may produce results like those shown in Figures 2 and 4.

We explored this possibility in the present experiment by having observers adjust the SOA until the two stimuli appeared simultaneous, or if simultaneity was not perceived, until the perceived asynchrony was minimized. The advantage of this procedure is that the points of greatest temporal uncertainty consist of direct estimates provided by the observers and are not inferred from the proportion of left-first, rightfirst, and simultaneous responses.

\section{Method}

Observers. Five observers participated in the experiment, the 2 authors and 3 others who were unaware of the hypothesis under investigation. All observers had participated in at least one of the previous experiments.

Design and procedure. The display and design were the same as that in Experiment 1. There were six conditions defined by the factorial combination of direction of attention (left, right, and center) and starting SOA (70 $\mathrm{ms}$ and $-70 \mathrm{~ms}$ ).

Within a session, conditions were tested in random order, but trials were blocked by condition. Each observer served in 10 sessions.

Observers adjusted the SOA in 5-ms steps, passing though the critical SOA as many times as they wished until they were confident about their final setting. If simultaneity was not clearly perceived, observers were instructed to search for the SOA at which the perceived asynchrony was minimal. If simultaneity was perceived over a range of SOAs, observers were instructed to adjust the SOA to the middle of this range. They used a double press of a button to signal completion of an adjustment.

\section{Results and Discussion}

Results are shown in Figure 6 with histograms. Each histogram shows the frequency (summed across subjects, sessions 
and starting SOA) with which each SOA was selected to be the point of simultaneity/minimal asynchrony. As in Experiment 1 , the histograms were constructed by first quantizing the SOA range into 20 -ms bins and then plotting the frequency with which the estimates fell into each bin as a percentage of the total.

As Figure 6 shows, direction of attention had large effects on the point of perceived simultaneity/minimal asynchrony. Importantly, because the points of greatest temporal uncertainty were not inferred from the proportion of right-first and left-first responses, the effects cannot be attributed to a bias to respond with the side to which attention was directed. The present results, combined with those of the previous experiment, provide strong evidence against a response-artifact explanation of the effects of directed attention.

The data were analyzed statistically with a 3 (attentional locus: left, right, and center) $\times 2$ (starting SOAs: 70 and -70 ) $\times 10$ (session) ANOVA. In general, the pattern of statistically significant effects was similar to that of Experiment 1. A significant main effect of attentional locus, $F(2,8)=20.6$, $p<.001$, indicates that the means of the histograms were different $(-24.1,21.3 \mathrm{~ms}$ and $-5.9 \mathrm{~ms}$ for the right-, left-, and center-attend conditions, respectively). The differences among the means were slightly smaller than those observed

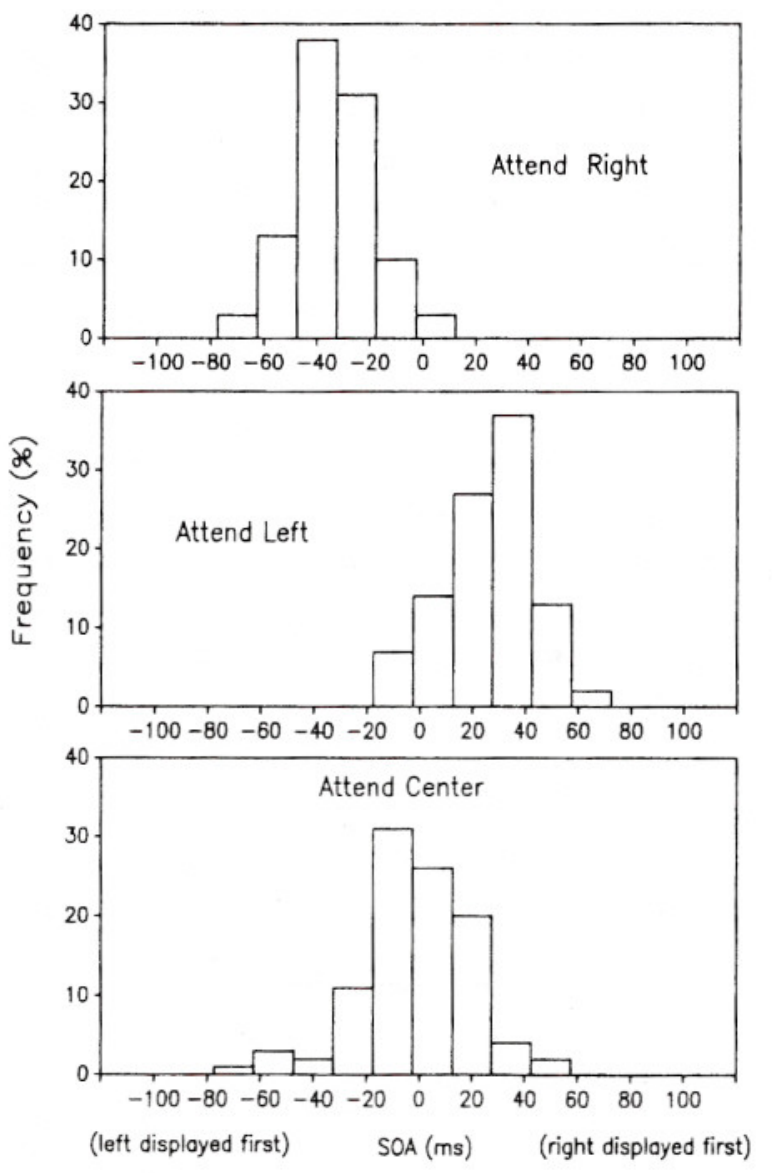

Figure 6. Frequency of simultaneity/minimal asynchrony responses across SOAs (Experiment 5). in Experiment 1 (compare Figures 2 and 6) because observers directed their attention to one location over a block of trials in the present experiment but varied the direction of attention from trial to trial in the previous experiments. Similar effects of blocking have been reported in previous work (Posner et al., 1980). The effect of starting SOA approached significance, $F(1,4)=5.5, p<.08$. The Starting SOA $\times$ Locus interaction was significant, $F(2,8)=25.2, p<.001$, indicating (as before) that the dispersion of the histograms was greater in the centerattend than in the left- and right-attend conditions.

\section{Experiment 6}

In Experiments 1-5 an indicator dot was placed under the to-be-attended stimulus location and remained on throughout the entire trial. An additional zoom (helper signal) was displayed to aid focusing of attention. A potential problem with these procedures is that they may increase the sensitivity of the retina and the visual system at the attended location in relation to the unattended location (e.g., by increasing the local level of light adaptation). These potential changes in sensitivity may cause the attended stimulus to be processed more rapidly, independent of any effects of attention. To rule out this explanation, we used a centrally presented arrow cue in the present experiment. The arrow cue was located beneath the central fixation marker and pointed either left, right, or up; it instructed the observer to attend to the left, to the right, or to the central marker box. An indicator dot and zoom (helper signal) were not presented. Because the arrow cue was always presented in the same central location, the attended and the unattended sides were physically identical. If the results of the present experiment concur with those from the previous experiments, an explanation that attributes the effects to changes in local sensitivity would be untenable. Instead, the results would support an attentional explanation.

\section{Method}

Observers. Five observers participated in the experiment, the 2 authors and 3 others who were unaware of the hypothesis under investigation. All but 1 observer had participated in previous experiments.

Design and procedure. The display, design, and procedures were the same as in Experiment 2, with the following exceptions. The indicator dot and the zoom (helper signal) were not presented. Instead, an arrow located $0.5^{\circ}$ below the central fixation marker was displayed throughout the trial. The arrow was $0.4^{\circ}$ long and $0.4^{\circ}$ wide. The stimulus dots were presented $250 \mathrm{~ms}$ after a trial was initiated by the observer. Each observer participated in six sessions, yielding 30 observations per condition.

\section{Results and Discussion}

Figure 7 shows a comparison of the present data obtained by using a central arrow cue (dashed lines) to those obtained by using peripheral cues (solid lines). The solid lines were taken from the baseline condition of Experiment 3.

As can be seen in Figure 7, the data from the two experiments are in close agreement, indicating that both types of 

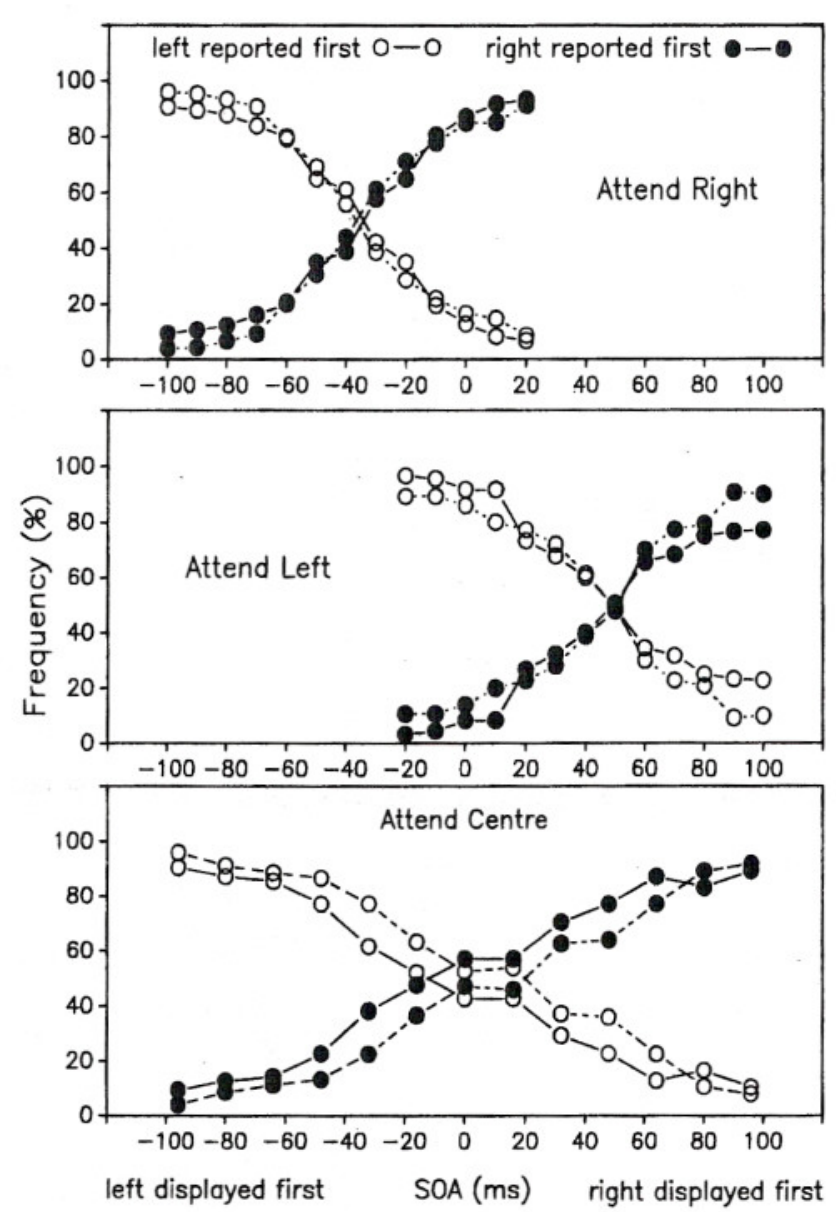

Figure 7. Frequency of left-first and right-first responses across SOAs with a central arrow cue (Experiment 6: dashed lines) and with peripheral cues (Experiment 3: solid lines).

attentional cues (central and peripheral) resulted in near-equal changes in perception of temporal order. These results show that the effects found in the present research cannot be attributed to changes in local visual sensitivity caused by the peripheral cues. We conclude that perception of temporal order is influenced by directed attention.

\section{General Discussion}

The purpose of the present research was to examine the assumption that directing attention influences the speed of transmission of information in the visual system. This was accomplished by integrating the standard dissociation technique (where the direction of gaze and the direction of attention are dissociated) with a task involving judgments of temporal order of two visual stimuli. In Experiments 1 and 2 it was established that directing attention influences the perception of temporal order. Experiments 3-6 extended the findings of the first two experiments while addressing various methodological issues.

The results of the present research can be summarized as follows. First, attended stimuli were perceived to occur before unattended stimuli even when the two were displayed at the same time. Second, in the center-attend condition there was a broader range of SOAs over which observers were unable to perceive the order of the two stimuli reliably. Third, in the left- and right-attend conditions, observers virtually never reported the two stimuli as occurring simultaneously. By contrast, in the center-attend condition, there was a large percentage of simultaneous responses, centered and evenly distributed around an SOA of $0 \mathrm{~ms}$. Fourth, these findings cannot be attributed to eye movements, to a bias to respond with the attended side, or to possible changes in local sensitivity because of the presence of peripheral cues.

We used the general threshold model as a framework to examine the effects of directed attention on temporal-order judgments. The model was able to predict the results of the center-attend condition but failed to explain the lack of simultaneous responses in the left- and right-attend conditions. One reason for this failure is that according to the general threshold model, perception of simultaneity should be equivalent to uncertainty about temporal order. This assumption was not supported by the present results: Given uncertainty about temporal order, observers did not necessarily perceive the two stimuli as being simultaneous. We conclude that judgments of simultaneity and judgments of temporal order are each based on different information. To account for these results, we propose a temporal-profile model that includes two comparators, one for simultaneity and one for temporal order.

\section{Temporal Profile Model}

General outline. A schematic of the temporal-profile model is presented in Figure 8. There are three major components in the model: a temporal-order comparator, a simultaneity comparator, and a decision mechanism. The temporal-order comparator computes a difference of its inputs. The simultaneity comparator computes an index of the degree of temporal overlap of its inputs. The outputs of the two comparators feed into a decision mechanism that generates a response. If only two response alternatives are available (left first and right first), the decision is based solely on the information from the temporal comparator. The role of attention in this model is to change the temporal profile of the responses that enter the comparators.

Temporal impulse responses. Input to both comparators is modeled after Roufs and Blommeart (1981) with the follow-

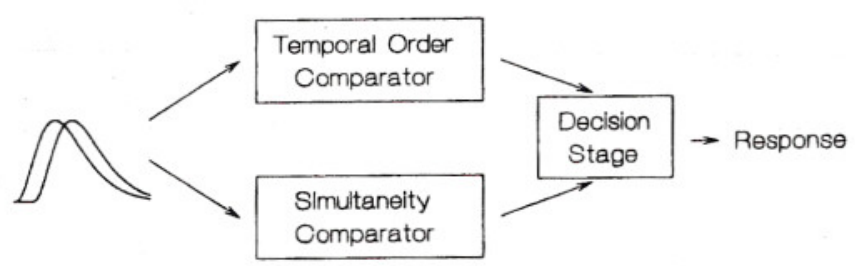

Figure 8. Schematic representation of the temporal-profile model. 
ing formula:

$$
f(t, b)=\left(.742 *(t / b)^{3} * \exp (t / b) *-1\right) .
$$

Formula (2) characterizes the temporal response of the visual system to a pulse of light and is referred to as the temporal impulse response function. We used Formula (2) for convenience, although any other formula describing the shape of the temporal impulse response function (e.g., Ikeda, 1986; Watson, 1986) may be substituted without affecting the predictions of the model. Parameter $t$ is time in $\mathrm{ms}, b$ is a free parameter, and .742 is a constant that normalizes the response, ensuring that the peak of the function is 1. Parameter $b$ controls the temporal profile of the function. With larger $b$ the function becomes broader, taking more time to reach a maximum and more time to subside. With smaller $b$ the response is more brisk, reaching a maximum more quickly and subsiding earlier. When two stimuli are displayed (as in the present experiments), there are two inputs to each comparator.

Temporal-order comparator. The comparator computes a difference of its inputs. ${ }^{2}$ Inputs are shown in the left column of Figure 9; outputs of the comparator are shown in the right
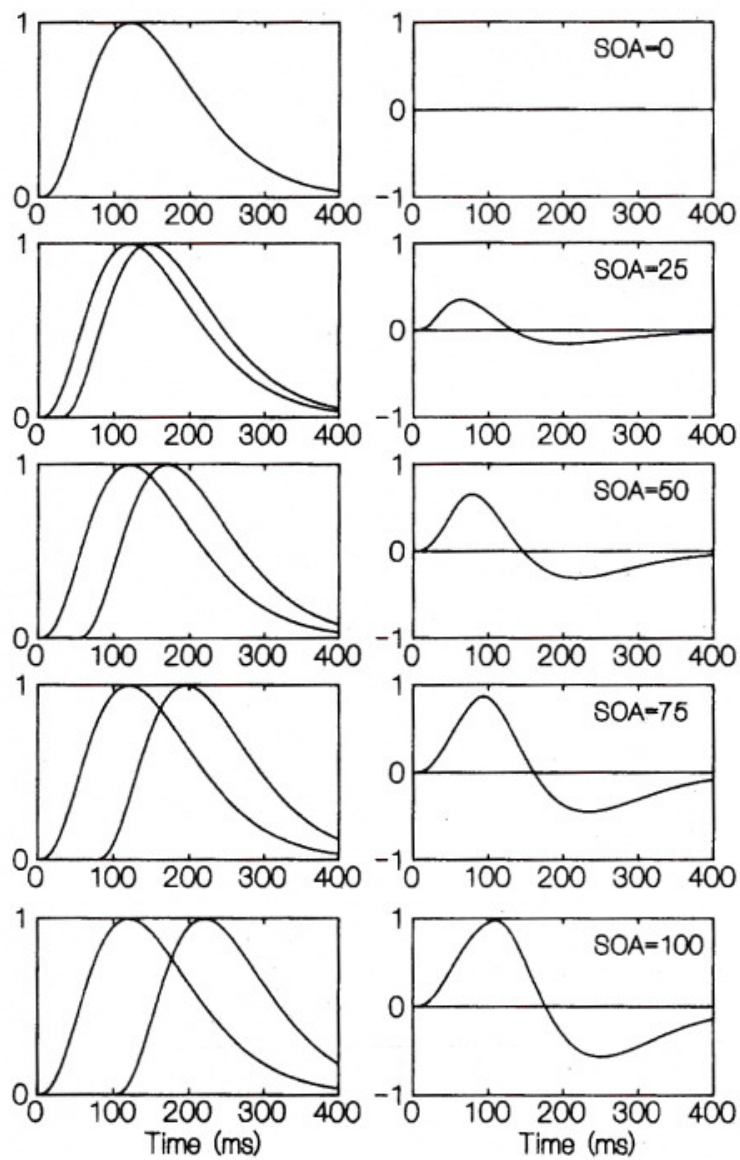

Figure 9. Hypothetical visual responses in the center-attend condition. (Left panels represent temporal impulse response functions. Right panels show the result of subtracting the corresponding impulse functions as would occur in the temporal comparator.) column of Figure 9. When stimuli are presented at an SOA of $0 \mathrm{~ms}$, the two responses overlap completely (top left panel of Figure 9). Thus, the difference as computed in the temporal-order comparator is zero (top right panel of Figure 9). As the SOA between the stimuli increases, the responses separate in time, and a nonzero difference results (as shown in the other panels in Figure 9).

The degree to which one stimulus precedes the other in time is reflected in the absolute value of the first peak in the difference profile. If the first stimulus precedes by a large SOA, the first peak in the difference profile will be large. In this case, observers would be likely to report the stimulus associated with the peak as occurring first. If the SOA is close to $0 \mathrm{~ms}$, then the peak in the difference profile corresponding to the first stimulus will be small, and observers will be less likely to report the stimulus associated with that peak as occurring first. The sign of the peak (positive or negative) indicates which stimulus occurred first.

Figure 9 represents responses in the visual system that would correspond to the center-attend condition. The transition from no peak in the difference profile ( $\mathrm{SOA}=0 \mathrm{~ms}$ ) to a large peak ( $\mathrm{SOA}=75 \mathrm{~ms}$ approximately) explains why certainty of temporal-order judgments increased with SOA in the center-attend condition. The two input responses (left panels, Figure 9) are equivalent because an equal amount of attention is allocated toward both stimulus locations.

Simultaneity comparator. It is assumed that judgments of simultaneity are based on a ratio of common versus uncommon areas of the two impulse responses. ${ }^{3}$ Observers are more likely to respond "simultaneous" if the area of the overlapping region of the impulse responses is large in relation to the nonoverlapping regions. At an SOA of $0 \mathrm{~ms}$, overlap is complete, yielding an infinite ratio. With greater SOA, the magnitude of the ratio decreases as the two responses separate. Thus, in the center-attend condition, judgments of simultaneity were maximal at an SOA of $0 \mathrm{~ms}$ (high ratio) and dropped with increasing SOA (low ratio).

Effects of attention. To explain the effects of directed attention on temporal order and simultaneity judgments, attention was assumed to cause the visual response to become more brisk. ${ }^{4}$ This effect can be modeled by decreasing the value of $b$ in Formula (2). We assumed a value of $b=12$ for the attended stimulus location and a value of $b=40$ for an unattended stimulus location. These values are plausible to the extent that Roufs and Blommeart (1981) estimated $b$ at 12.66 under conditions in which the direction of gaze and the direction of attention coincided.

Figure 10 shows situations in which attention is directed to one stimulus location and away from the other location, as

\footnotetext{
${ }^{2}$ The notion of computing a difference of temporal signals is inspired by Marr's (1982, p. 62) treatment of spatial signals.

${ }^{3}$ The simultaneity comparator is based on work in visible persistence (Groner, Bischof, \& DiLollo, 1988).

${ }^{4}$ It is possible that there are concurrent effects in which the signal corresponding to the attended stimulus becomes more brisk, and the signal corresponding to the unattended stimulus becomes less brisk. The data from the present experiments, however, may be modeled by making changes to the profiles of the attended signal alone.
} 

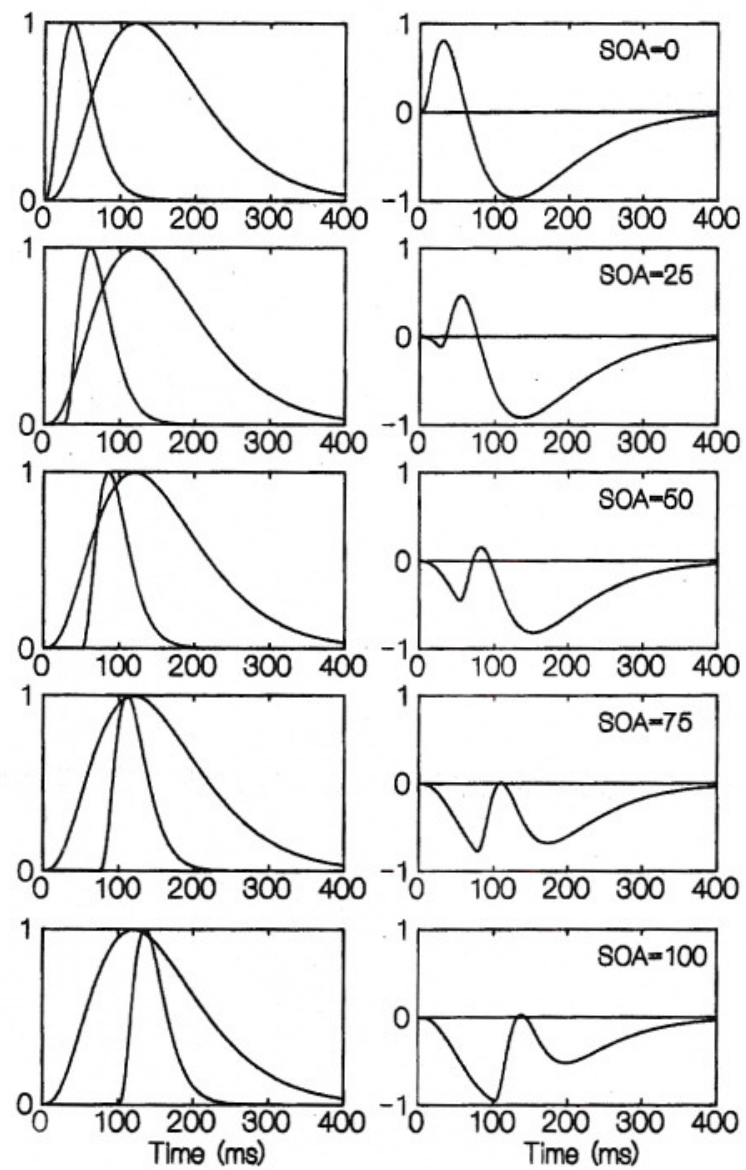

Figure 10. Hypothetical visual responses in the left- and right-attend conditions. (Left panels represent temporal impulse response functions. The narrower function corresponds to the attended response, and the broader function corresponds to the unattended response. Right panels show the result of subtracting the corresponding impulse functions as would occur in the temporal comparator.)

occurred in the left- and right-attend conditions. The narrower function represents an impulse response at the attended location, whereas the broader function represents an impulse response at the unattended location. At an SOA of $0 \mathrm{~ms}$, the function for the attended stimulus precedes the function for the unattended stimulus (top left panel, Figure 10). In the difference profile (top right panel, Figure 10), the order of the responses is reflected in a strong peak corresponding to the onset of the attended stimulus. Accordingly, observers consistently reported that the attended stimulus appeared to precede the unattended stimulus in the left- and right-attend conditions at an SOA of $0 \mathrm{~ms}$.

Displaying the unattended stimulus prior to the attended stimulus increased the degree to which the unattended response preceded the attended response. The first peak in the difference profile associated with the unattended stimulus (negative lobe) grows as the temporal lead of the unattended stimulus is increased. This explains why observers reported that the unattended stimulus appeared to occur first more frequently as its temporal lead increased.
In the present research, the steeper slopes and the lesser dispersion of the distributions in the left- and right-attend conditions (Figure 2) can be readily explained by reference to Figures 9 and 10. In Figure 10 the transition from positive lobe preceding to negative lobe preceding occurred within a range of about $75 \mathrm{~ms}$. Hence the transition from one stimulus appearing to occur first to the other stimulus appearing to occur first ought to be complete within about $75 \mathrm{~ms}$. This agrees with the results in the left- and right-attend conditions, in which the transition from left-first to right-first judgments occurred within about $75 \mathrm{~ms}$.

In Figure 9, which represents the center-attend condition, only half of the transition is shown, from the point of simultaneity to the point where one stimulus precedes the other. The entire transition from positive lobe preceding to negative lobe preceding would take about $150 \mathrm{~ms}(2 \times 75 \mathrm{~ms})$. This agrees with the results in the center-attend condition, in which the transition from left-first judgments to right-first judgments occurred within about $150 \mathrm{~ms}$.

The temporal-profile model can also account for the absence of simultaneous perceptions in the left- and right-attend conditions. As illustrated in Figure 10, the temporal impulse responses in the simultaneity comparator would never overlap completely at any SOA. Hence a strong signal for simultaneity wound never be present. In contrast, a strong signal for asynchrony from the temporal-order comparator would always be available, as shown in the right panel of Figure 10. Consistent with the model, perceptions of asynchrony were dominant at all SOAs in the left- and right-attend conditions.

\section{Conclusions}

Spotlight (LaBerge, 1983; Posner, et al., 1980), zoom (Eriksen \& Yeh, 1985), and gradient (LaBerge \& Brown, 1989) models have been successful in accounting for the effects of directed attention across a wide variety of research domains. The temporal-profile model is consistent with these models to the extent that attention is assumed to enhance the transmission speed of visual information in the sensory-perceptual system. The temporal-profile model extends the earlier models by providing an explicit characterization of the temporal changes that arise in early visual processing as a result of directed attention. Effects of directed attention are represented in the temporal-profile model as a change in one parameter of the temporal impulse response function.

The present research demonstrates that temporal modulation is one important consequence of attentional allocation. It is important to note, however, that temporal modulation is likely not the only consequence of attentional allocation; there must be other modulating influences as well. The nature of the relationship between temporal modulation and other attentional operators is an important area for future research.

There are numerous basic and applied questions that are stimulated by the temporal-profile model. For example, the apparent duration of the attended and unattended stimuli should differ with the duration of the unattended stimulus appearing to be longer because of its broader temporal profile. In the applied domain, the increase in processing efficiency at attended locations would be important when viewing scenes 
that contained rapid change. Specifically, the modulating effect of attention, as described by the temporal-profile model, may have implications for the perception of motion, flicker, and other visual stimuli that contain rapid change. In this view, the model may have implications for the design of video systems that use temporal sampling (e.g., television). For example, the perceptibility of flicker and temporal aliasing (jerky motion) may be greater at attended locations than at unattended locations.

\section{References}

Allan, L. G. (1975). The relationship between judgments of successiveness and judgments of order. Perception \& Psychophysics, 18, 29-36.

Corwin, T. R., \& Boynton, R. M. (1968). Transitivity of visual judgments of simultaneity. Journal of Experimental Psychology, 78, 560-568.

Eriksen, C. W., \& St. James, J. D. (1986). Visual attention within and around the field of focal attention: A zoom lens model. Perception \& Psychophysics, 40, 225-240.

Eriksen, C. W., \& Yeh, Y. (1985). Allocation of attention in the visual field. Journal of Experimental Psychology: Human Perception and Performance, 11, 583-597.

Groner, M. T., Bischof, W. F., \& Di Lollo, V. (1988). A model of visible persistence and temporal integration. Spatial Vision, 3, 293304.

Ikeda, I. (1986). Temporal impulse response. Vision Research, 26, 1431-1440.

LaBerge, D. (1983). The spatial extent of attention to letters and words. Journal of Experimental Psychology: Human Perception and Performance, 9, 371-379.

LaBerge, D., \& Brown, V. (1989). Theory of attentional operations in shape identification. Psychological Review, 96, 101-124.

Marr, D. (1982). Vision. San Francisco: Freeman.

Needham, J. G. (1936). Some conditions of prior entry. Journal of General Psychology, 14, 226-240.
Posner, M. I., Snyder, C. R. R., \& Davidson, J. B. (1980). Attention and the detection of signals. Journal of Experimental Psychology: General, 109, 160-174.

Roufs, J. A. J., \& Blommaert, F. J. J. (1981). Temporal impulse and step responses of the human eye obtained psychophysically by means of a drift-correcting perturbation technique. Vision Research, 21, 1203-1221.

Rutschmann, R. (1966). Perception of temporal order and relative visual latency. Science, 152, 1099-1101.

Sekuler, R. (1976). Seeing and the nick in time. In M. H. Siegel \& H. P. Zeigler (Ed.), Psychological research: The inside story (pp. 176-197). New York: Harper \& Row.

Sekuler, R., Tynan, P., \& Levinson, E. (1973). Visual temporal order: A new illusion. Science, 180, 210-212.

Shaw, M. L. (1978). A capacity allocation model for reaction time. Journal of Experimental Psychology: Human Perception and Performance, 4, 586-598.

Sternberg, S., \& Knoll, R. L. (1973). The perception of temporal order: Further issues and a general model. In S. Kornblum (Ed.), Attention and performance IV (pp. 629-685). New York: Academic Press.

Sternberg, S., Knoli, R. L., \& Gates, B. A. (1971, November). Prior entry reexamined: Effect of attentional bias on order perception. Paper presented at the meeting of the Psychonomic Society, St. Louis, Missouri.

Taylor, M. M., \& Creelman, C. D. (1967). PEST: Efficiency estimates on probability functions. Journal of the Acoustical Society of America, 41, 782-787.

Ulrich, R. (1987). Threshold models of temporal-order judgments evaluated by a ternary response task. Perception \& Psychophysics, 42, 224-239.

Watson, A. B. (1986). Temporal sensitivity. In K. R. Boff, L. Kaufman, \& J. P. Thomas (Ed.), Handbook of perception and human Performance: Sensory processes and perception (Vol 1, pp. 6-16-43). New York: Wiley.

Received January 11, 1990

Revision received August 10, 1990

Accepted October 9, 1990 